\title{
Fascin is involved in the chemotherapeutic resistance of breast cancer cells predominantly via the PI3K/Akt pathway
}

\author{
H Ghebeh ${ }^{1}$, S Al-Khaldi ${ }^{2}$, S Olabi ${ }^{1}$, A Al-Dhfyan ${ }^{1}$, F Al-Mohanna ${ }^{3}$, R Barnawi ${ }^{1}$, A Tulbah ${ }^{4}$, T Al-Tweigeri ${ }^{5}$, \\ D Ajarim ${ }^{5}$ and M Al-Alwan*,1
}

${ }^{1}$ Stem Cell and Tissue Re-Engineering Program, King Faisal Specialist Hospital and Research Centre, MBC: 03-99, P.O. Box: 3354, Riyadh 11211, Saudi Arabia; ${ }^{2}$ The Joint Center for Genomics Research, King Abdulaziz City for Sciences and Technology, Riyadh, Saudi Arabia; ${ }^{3}$ Department of Comparative Medicine, King Faisal Specialist Hospital and Research Centre, MBC: 03-99, P.O. Box: 3354, Riyadh 11211, Saudi Arabia; ${ }^{4}$ Department of Pathology and Laboratory Medicine, King Faisal Specialist Hospital and Research Centre, MBC: 03-99, P.O. Box: 3354, Riyadh 11211, Saudi Arabia and ${ }^{5}$ Department of Oncology, King Faisal Specialist Hospital and Research Centre, MBC: 03-99, P.O. Box: 3354, Riyadh 11211, Saudi Arabia

Background: A major therapeutic challenge for breast cancer is the ability of cancer cells to evade killing of conventional chemotherapeutic agents. We have recently reported the actin-bundling protein (fascin) as a major regulator of breast cancer metastasis and survival.

Methods: Survival of breast cancer patients that received chemotherapy and xenograft tumour model was used to assess the effect of chemotherapy on fascin-positive and -negative breast cancer cells. Molecular and cellular assays were used to gain in-depth understanding of the relationship between fascin and chemoresistance.

Results: We showed a significant correlation between fascin expression and shorter survival in breast cancer patients who received chemotherapy. In xenograft experiments, fascin-positive cancer cells displayed significantly more resistance to chemotherapymediated apoptotic cell death than fascin-negative counterparts. This increased chemoresistance was at least partially mediated through PI3K/Akt signalling, and was paralleled by increased FAK phosphorylation, enhanced expression of the inhibitor of apoptosis proteins (XIAP and Livin) and suppression of the proapoptotic markers (caspase 9, caspase 3 and PARP).

Conclusions: This is the first report to demonstrate fascin involvement in breast cancer chemotherapeutic resistance, supporting the development of fascin-targeting drugs for better treatment of chemoresistance breast cancer.

Recent years have witnessed progress in the treatment of breast cancer, which has significantly improved the survival of patients (Gladney et al, 1999; Balduzzi et al, 2013). Despite this progress, successful treatment of metastatic breast cancer remained considerably low (Khope et al, 1988; Dellapasqua et al, 2014). Failure to achieve greater therapeutic success has been attributed to the ability of cancer cells to evade killing of standard chemotherapy. The drug-resistant cell population upon activation may gain the ability to re-grow leading to tumour relapse and metastasis.
Therefore, a better treatment strategy should focus on understanding the underlying mechanisms that regulate the cancer cell resistance to chemotherapy and target those pathways. Such therapeutic intervention would have a great impact not only on the success rate of treatment, but also on reducing the chances of tumour relapse.

It is well established that actin cytoskeletal network has a central role in many important biological processes including cell motility, which is one of the cellular mechanisms that regulate tumour

*Correspondence: Dr M Al-Alwan; E-mail: MAlwan@kfshrc.edu.sa

Received 6 May 2014; revised 14 July 2014; accepted 16 July 2014; published online 12 August 2014 
invasiveness and metastasis (Jiang et al, 2009). Fascin is an actinbundling cytoskeletal protein with highly restricted expression in specialized normal cells (Duh et al, 1994; Mosialos et al, 1994, 1996; Pinkus et al, 1997). However, many transformed cells including breast cancer cells also expressed fascin (Khwaja et al, 1997). Interestingly, fascin expression in malignancies correlates with the clinical aggressiveness of tumours and metastasis. We have recently reported that fascin confers breast cancer metastasis by regulating metastasis-associated genes via upregulation of the NF- $\kappa$ B pathway and suppression of the Breast Cancer Metastasis Suppressor 1 (BRMS1) gene (Al-Alwan et al, 2011). Metastatic cells are well known to be chemoresistant. Whether fascin regulation of metastasis-associated genes is associated with increased resistance of metastatic cells to apoptotic cell death has not been previously investigated.

It has been reported that chemotherapy kills target cells largely by the induction of intrinsic pathway of apoptosis (reviewed in Kaushal et al (2004); Minotti et al (2004)), which is initiated by the activation of caspase 9 (Eliceiri et al, 2002). Upon initiation of this process, caspase 9 activates the executioner of apoptosis caspases 3 and 7, which in turn cleave several cellular targets especially Poly (ADPribose) polymerase (PARP) that ultimately facilitate cellular death upon cleavage. The apoptotic cell death is a tightly regulated process by another set of inhibitor of apoptosis protein (IAP) family. For example, X-linked inhibitor of apoptosis protein (XIAP) is a member of the IAP family that has been shown to have a direct inhibitory effect on caspase 9 (Dan et al, 2004). Most importantly, XIAP was reported to be a downstream target of the phosphatidylinositol 3-kinase (PI3K)/Akt pathway. Many cancer cells evade apoptosis through the activation of PI3K/Akt pathway and mutation or downregulation mutation of tumour suppressor gene PTEN, a negative regulator of the PI3K pathway (West et al, 2002; Fresno Vara et al, 2004; Zhang \& Yu, 2010; Dituri et al, 2011).

In this study, we have demonstrated a strong relationship between fascin expression and resistance to chemotherapy. In xenograft tumour mouse model, fascin-positive cells were more resistant to chemotherapy-mediated apoptotic cell death than their fascinnegative counterpart. Interestingly, fascin-positive cells that were exposed to chemotherapy showed enhanced PI3K/Akt, suppressed PTEN and enhanced IAP (XIAP and Livin) expression. Inversely, chemotherapy-exposed cells showed decreased levels of caspase 9, caspase 3 and PARP expression in fascin-positive cells compared with fascin-negative counterparts. Our results demonstrate important roles for fascin in regulating breast cancer cell sensitivity to the neoadjuvant chemotherapy-mediated apoptotic cell death.

\section{MATERIALS AND METHODS}

Patients selection. Breast cancer specimens were obtained from archived paraffin-embedded tumours of 139 patients including the 71 patients reported in our previous study (Al-Alwan et al, 2011) who were seeking treatments and had to undergo surgery (breast conservative surgery or total mastectomy) at King Faisal Specialist Hospital and Research Centre (KFSH\&RC) from 2003 to 2006. A waiver of consent were obtained from the institutional board under RAC\# 2060 016).

Breast cancer patients were treated with KFSH\&RC standard protocol consisting of four cycles of a combination of doxorubicin and cyclophosphamide followed by four cycles of docetaxel. Sixteen patients (of which five (31.25\%) were positive for fascin) received cisplatin along with docetaxel as a part of clinical trial (AlTweigeri et al, 2010). All patients having tumours positive for her2 were treated with trastuzumab. Relapsing patients were treated with capecitabine, gemcitabine, paclitaxel and/or vinorelbine. All metastatic patients with Her2 + tumours received trastuzumab.
Animal and cell lines. Nude mice were from Jackson Immuno Research Laboratories, Bar Harbour, ME, USA. The animals were housed and maintained in accordance with the protocols approved by the Animal Care and Use Committee of the King Faisal Specialist Hospital and Research Centre.

The human breast cancer cell lines MDA-MB-231 (HTB-26), SK-BR-3 (HTB-30) and T47-D (HTB-20) were purchased from ATCC (Manassas, VA, USA). The stable fascin knockdown (ShFascin) or control (ShCon) as well as the transient fascin knockdown (SiFascin) or control (ShCon) MDA-MB-231 cells were generated and described previously (Al-Alwan et al, 2011). The stable fascin-expressing T47-D cells were generated and described previously (Al-Alwan et al, 2011). Cells were grown in DMEM containing $10 \%$ fetal bovine serum (Invitrogen, Grand Island, NY, USA), $200 \mathrm{~mm}$ L-glutamine (Invitrogen) and antibiotic-antimycotic liquid (Invitrogen) at $37^{\circ} \mathrm{C}$ in a $5 \% \mathrm{CO}_{2}$ humidified atmosphere. Cell authentication confirmed the identity and purity of the used cells in reference to the eight core STR markers, which are recommended by ATCC, and carried out using the Promega PowerPlex Systems (Madison, WI, USA).

Antibodies and reagents. The anti-fascin $\mathrm{mAb}$ (mouse IgG1) was purchased from Dako (Carpinteria, CA, USA). The APCconjugated goat ant-mouse IgG1 secondary Ab was purchased from Jackson ImmunoResearch Laboratories. The rabbit antibodies to detect c-IAP1, c-IAP2, Survivin, XIAP, Livin, cleaved caspase 9, AKT, p-AKT and PTEN, FAK, p-FAK and HRP-labelled anti-rabbit secondary antibody were obtained from Cell Signaling (Beverly, MA, USA). The APC-labelled anti-active form caspase 3 and APC-Cy7 labelled anti-cleaved form PARP antibodies were from BD Biosciences (San Jose, CA, USA). The GAPDH and $\beta$-actin antibodies were obtained from Santa Cruz (Dallas, TX, USA). The secondary anti-mouse IgG1 HRP-conjugated and anti-goat IgG HRP-conjugated were from Southern Biotech (Birmingham, AL, USA) and Promega, respectively. The remaining antibodies were from BD Biosciences. The Vybrant apoptosis assay kit was purchased from Molecular Probes, Grand Island, NY, USA. The doxorubicin was purchased from Sigma, St Louis, MO, USA and the docetaxel (taxotere) was from Aventis Pharma, Dagenham Essex, UK.

Flow cytometry. Cell permeabilization and fascin staining were performed as previously described (Al-Alwan et al, 2011). Fluorescence was analysed on a total of $10^{4}$ cells per sample using a flow cytometer (LSR I; Becton Dickinson, Mountain View, CA, USA).

Apoptosis. Vybrant apoptosis assay kit (V13241; Life Technologies, Grand Island, NY, USA) was used to evaluate cell viability as per the manufacturer's recommendation. Briefly, both adherent and floating cells were collected after different time points of treatment with drugs. DAPI (4',6-diamidino-2-phenylindole) was used as a viability dye to avoid interference from doxorubicin natural florescence at the PE channel. Fluorescence was analysed on a total of $10^{4}$ cells per sample using a flow cytometer and cells were considered viable if they are double negative for Annexin V and DAPI. In some experiments, cells were also co-stained with anti-cleaved PARP ( $5 \mu \mathrm{l}$ per test) and anti-active form caspase 3 ( $5 \mu$ l per test) antibodies in combination with Annexin $\mathrm{V}$ and DAPI.

Western blot. Cells were harvested and washed with cold PBS before being lysed using RIPA buffer containing protease inhibitors (leupeptin $50 \mu \mathrm{g} \mathrm{ml}^{-1}$, pepstatin $20 \mu \mathrm{g} \mathrm{ml}^{-1}$, aprotitin $0.1 \mu \mathrm{g} \mathrm{ml}^{-1}$, phenylmethylsulfonyl fluoride $\left.174 \mu \mathrm{g} \mathrm{ml}^{-1}\right)$. The cells were incubated with the lysis buffer for $2 \mathrm{~h}$, vortexed every $10 \mathrm{~min}$ before centrifugation at 14000 r.p.m. at $4{ }^{\circ} \mathrm{C}$ for $15 \mathrm{~min}$. The protein concentration was determined using Bradford Assay (Bio-Rad, Hercules, CA, USA). $20 \mu \mathrm{g}$ of total cell lysate 
were loaded onto 10\% SDS-PAGE gel and transferred using iBlot pre-made nitrocellulose membrane and dry transfer system (Bay Cities Tool \& Supply Inc., Newark, CA, USA). Following the transfer, membranes were incubated in $5 \%(\mathrm{w} / \mathrm{v})$ skimmed milk in PBS containing $0.05 \%$ Tween-20 (PBST) to block nonspecific binding. Blots were then incubated with primary antibodies overnight at $4{ }^{\circ} \mathrm{C}$, washed with PBST four times and incubated with HRP-secondary antibody for $1 \mathrm{~h}$. Chemiluminescence Super Signal System (Thermo Scientific, Waltham, MA, USA) was used for subsequent detection of bound antibodies and bands were detected using ImageQuant LAS4010 Biomolecular Imager (GE Healthcare, Pittsburgh, PA, USA). Bands corresponding to the right size of the detected proteins were then semi-quantified using Quantity One program (Bio-Rad). Blots shown are representative of a minimum of three separate experiments.

Immunohistochemistry. Staining of formalin-fixed, paraffinembedded breast cancer samples (139 cases that have received chemotherapy) was performed as previously described (Ghebeh et al, 2006). Briefly, after deparaffinization and rehydration, antigen retrieval (Dako) was used before blocking endogenous peroxidase for $15 \mathrm{~min}$ with $3 \%$ hydrogen peroxide (Sigma) in methanol. Sections were then blocked with $10 \%$ goat serum (Sigma) for $60 \mathrm{~min}$, followed by the addition of a primary antihuman fascin antibody (1/200) overnight at $4{ }^{\circ} \mathrm{C}$. After washing, sections were incubated with labelled Polymer (EnVision ${ }^{+}$antimouse) HRP detection kit (Dako) for $30 \mathrm{~min}$ at room temperature. The HRP was detected using DAB substrate (Novocastra, Buffalo Grove, IL, USA) for $4 \mathrm{~min}$ and the sections were counterstained for 1 min with Harris hematoxylin (Acros Organics, Pittsburgh, PA, USA). Slides were washed with distilled water, dried and coverslipped using permanent mount (Novocastra). The intensity of staining and the percentages of fascin-positive cells were evaluated by two independent pathologists who had no prior knowledge of patient details.

Xenograft assay. Female nude mice (10 per group) at 6-8 weeks of age were injected subcutaneously into the right flank with $4 \times 10^{6}$ (suspended in $200 \mu \mathrm{l}$ of PBS:matrigel at $1: 1$ ratio) of fascin-positive (ShCon) and -negative (ShFascin) MDA-MB-231 breast cancer cells. The xenografts were monitored until the tumour volumes reached $\sim 100 \mathrm{~mm}^{3}$, each group was randomised into two subgroups that were either left untreated or received intraperitoneal injections of doxorubicin $\left(4 \mathrm{mg} \mathrm{kg}^{-1}\right)$ every 5 days (three cycles) as previously described by Hirsch et al (2009). The tumour volume was measured with a calliper using the following formula (length $\times$ width $\times$ height). All animal experiments were conducted in accordance with the protocols approved by the Animal Care and Use Committee of the King Faisal Specialist Hospital and Research Centre.

Statistical analysis. The significance (0.05) of relationship between fascin expression and the patient's clinicopathological parameters was assessed using Fisher's exact test. The software package JUMP (SAS Institute, Cary, NC, USA) was used for these analyses.

Other statistical significance was assessed using a one-way ANOVA (GraphPad InStat, San Diego, CA, USA). Wherever stated, NS denotes a $P$-value $>0.05$, while ${ }^{*}$ indicate $P$-values $<0.05$.

\section{RESULTS}

Fascin expression is associated with shorter survival in chemotherapy-treated breast cancer patients. We have previously reported a significant correlation between fascin expression in breast cancer patients and shorter disease-free survival (Al-Alwan et al, 2011). However, the correlation between fascin expression and overall survival in those patients did not reach statistical significance. Interestingly, the overall survival reached statistical significance $(P=0.0247)$ and the disease-free survival became even more significant $(P<0.001)$ when fascin expression was correlated only with patients that were exposed to prior chemotherapy (data not shown). While the sample size in the previous study was small (40 out of 71 cases were exposed to prior chemotherapy), the results implied a link between fascin expression and shorter survival in chemo-treated breast cancer patients. To further confirm this link on larger group size, we added 99 more cases that were also diagnosed with invasive ductal carcinoma and received neoadjuvant therapy and analysed their 10 -year survival. As we have previously reported, only 48 (35.29\%) of the total 139 breast cancer cases demonstrated fascin-positive tumour. In addition, there was a significant correlation between fascin expression in tumour and clinicopathological parameters including high histological grade, larger tumour size, negative oestrogen and progesterone receptors (Supplementary Table 1). Importantly, fascin-positive breast cancer patients who received neoadjuvant therapy correlated significantly with decreased disease-free survival $(P=0.0098)$ (Figure 1A) and overall survival $(P=0.0026$; Figure 1B). The shorter survival of chemotherapy recipient fascin-positive patients further supports a potential role for fascin in regulating chemotherapeutic resistance, a step that is widely believed to contribute to tumour relapse and metastasis.

Fascin-positive xenograft tumour is more resistant to the chemotherapeutic agent doxorubicin. To evaluate if fascin expression in breast cancer cells can directly contribute to their chemotherapy resistance, we used the tumorigenic and fascinpositive MDA-MB-231 breast cancer cells in a xenograft tumour model (Rochefort et al, 1998; Tong et al, 1999; Naffar-Abu-Amara
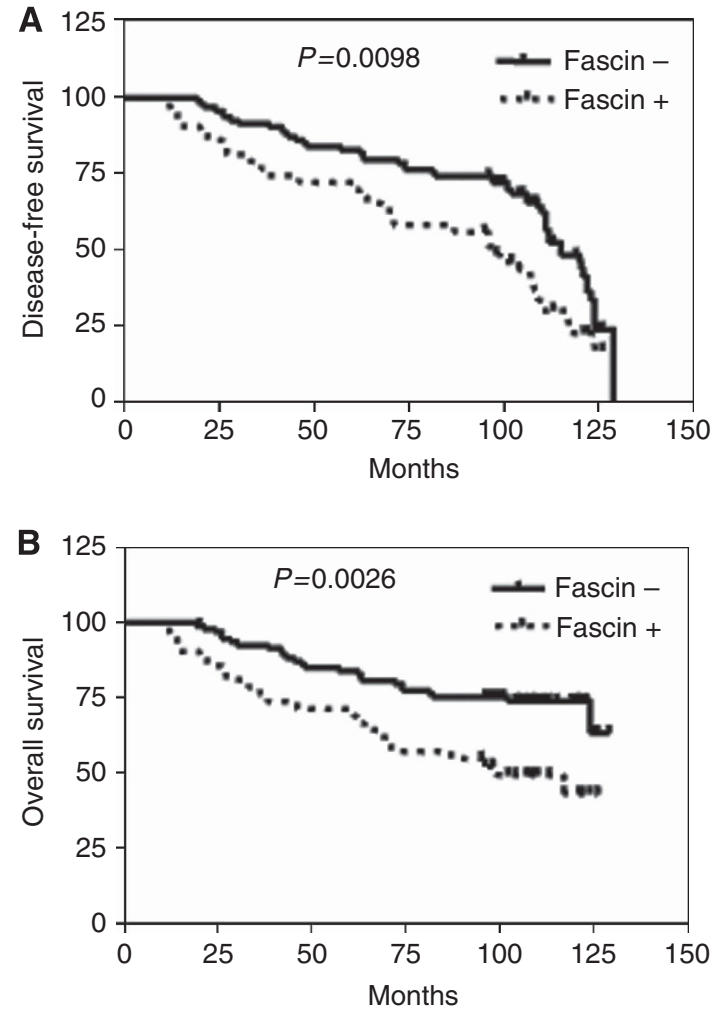

Figure 1. Shorter survival in chemo-treated human patients with fascin-positive tumour. (A and B) Kaplan-Meier survival curves showing survival in 139 patients who received chemotherapy blotted in relation to fascin expression. Survival was reported by oncologist and curves showing decreased disease-free (A) or overall (B) survival in fascinpositive patients that received chemotherapy. 
et al, 2008). Fascin expression was silenced using fascin-specific ShRNA (ShFascin) or scrambled control ShRNA (ShCon) as previously described (Al-Alwan et al, 2011). While both fascinpositive and -negative cells formed tumours (Figure 2A), the size of the tumour formed by fascin-negative cells were slightly larger (Figure 2B and C), although no difference in cell proliferation was observed when cells were grown in vitro (Al-Alwan et al, 2011). After 20 days of the tumour inoculation, the size of the fascinpositive and -negative tumour in the untreated group was doubling every 5 days. Upon treatment with doxorubicin (the mainstay of breast cancer chemotherapy), the size of fascin-negative tumour was significantly reduced (following the three cycles of treatment) in comparison with their fascin-positive counterpart. These findings suggest a direct correlation between fascin expression in breast cancer cells and increased resistance to chemotherapy.

It has been previously reported that chemotherapy target cancer cells via different mechanisms that ultimately lead to cell death by apoptosis (Kaushal et al, 2004; Minotti et al, 2004). To detect if doxorubicin-mediated cell death via apoptosis, single-cell suspensions were prepared from tumour xenografts excised from treated animal and co-stained for Annexin $\mathrm{V}$ and DAPI. Interestingly, even after more than 2 weeks of stopping the chemotherapy, more apoptotic cell death was observed in cells that were collected from doxorubicin-treated fascin-negative tumour compared with the ones collected from fascin-positive tumour counterpart (Figure 2D). Our data strongly suggest fascin involvement in regulating breast cancer chemoresistance at least in part via regulation of the apoptotic pathway.

Fascin-positive breast cancer cells is more resistant to chemotherapy-mediated apoptosis. To dissect the underlying mechanism of fascin regulation of breast cancer chemoresistance, we exposed the above-mentioned fascin-positive and -negative MDA-MB-231 cells to chemotherapy and measured their

A
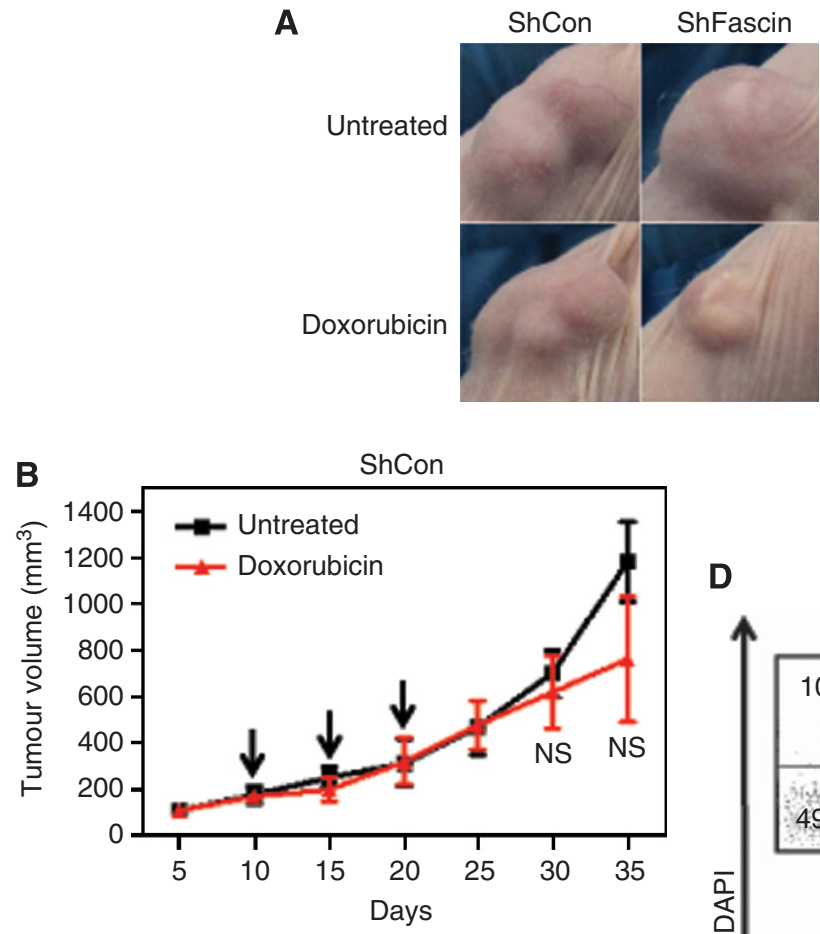

D
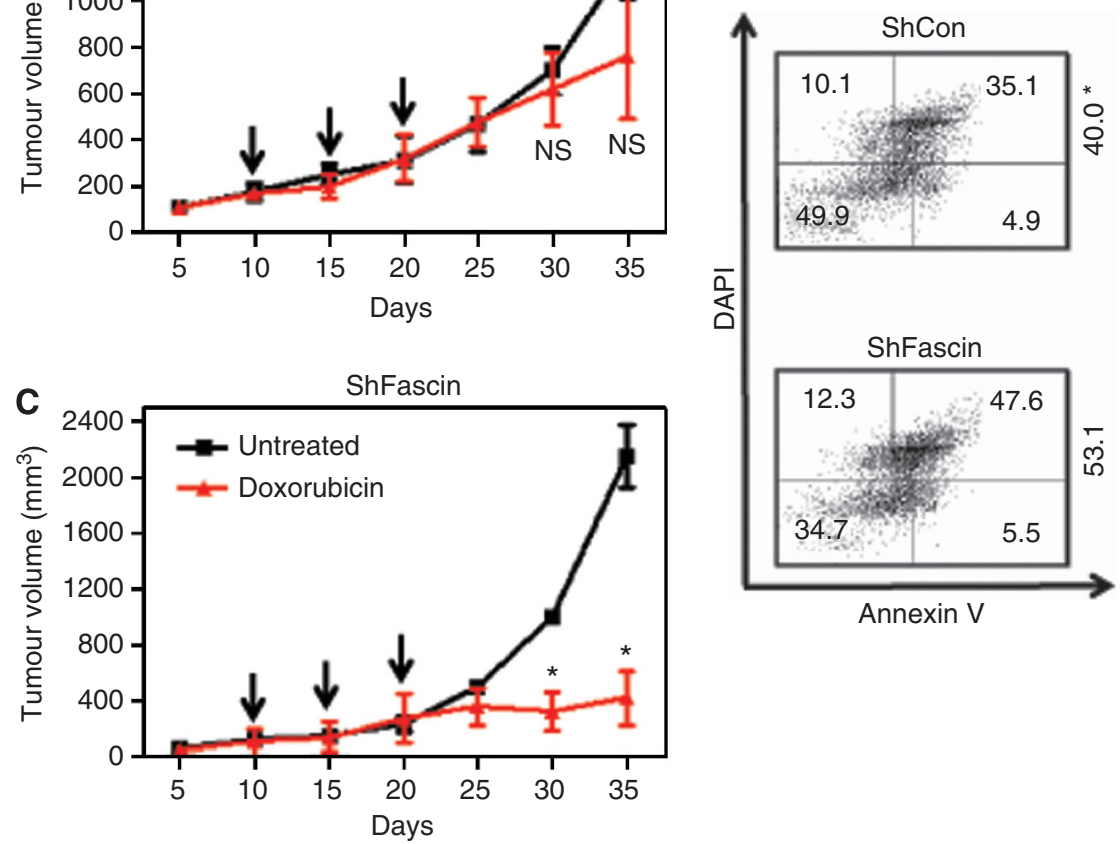

Figure 2. Loss of fascin expression sensitised breast cancer cells to chemotherapy in xenograft tumour model. (A-D) Fascin-positive (ShCon) and negative (ShFascin) MDA-MB-231 breast cancer cells were injected into nude mice as described in the Materials and Methods. The tumour volumes were measured following treatment with or without three cycles of doxorubicin. (A) Representative images showing tumour formed in nude mice after injection with ShCon or ShFascin cells. (B) Show mean \pm s.d. Tumour volumes in nude mice injected with ShCon cells and (C) show mean \pm s.d. tumour volumes in nude mice injected with ShFascin cells. The arrows indicate the time of doxorubicin injection. (D) ShCon (top) or ShFascin (bottom) xenograft tumours were collected from mice that received doxorubicin treatment at the end of the experiment and single-cell suspensions were prepared. Apoptotic cell death was detected by Annexin V and DAPI staining as described in the Materials and Methods.

* Numbers on the right of the dot plot represent the total percentage of apoptosis $(\mathrm{Q} 2+\mathrm{Q} 4)$. 

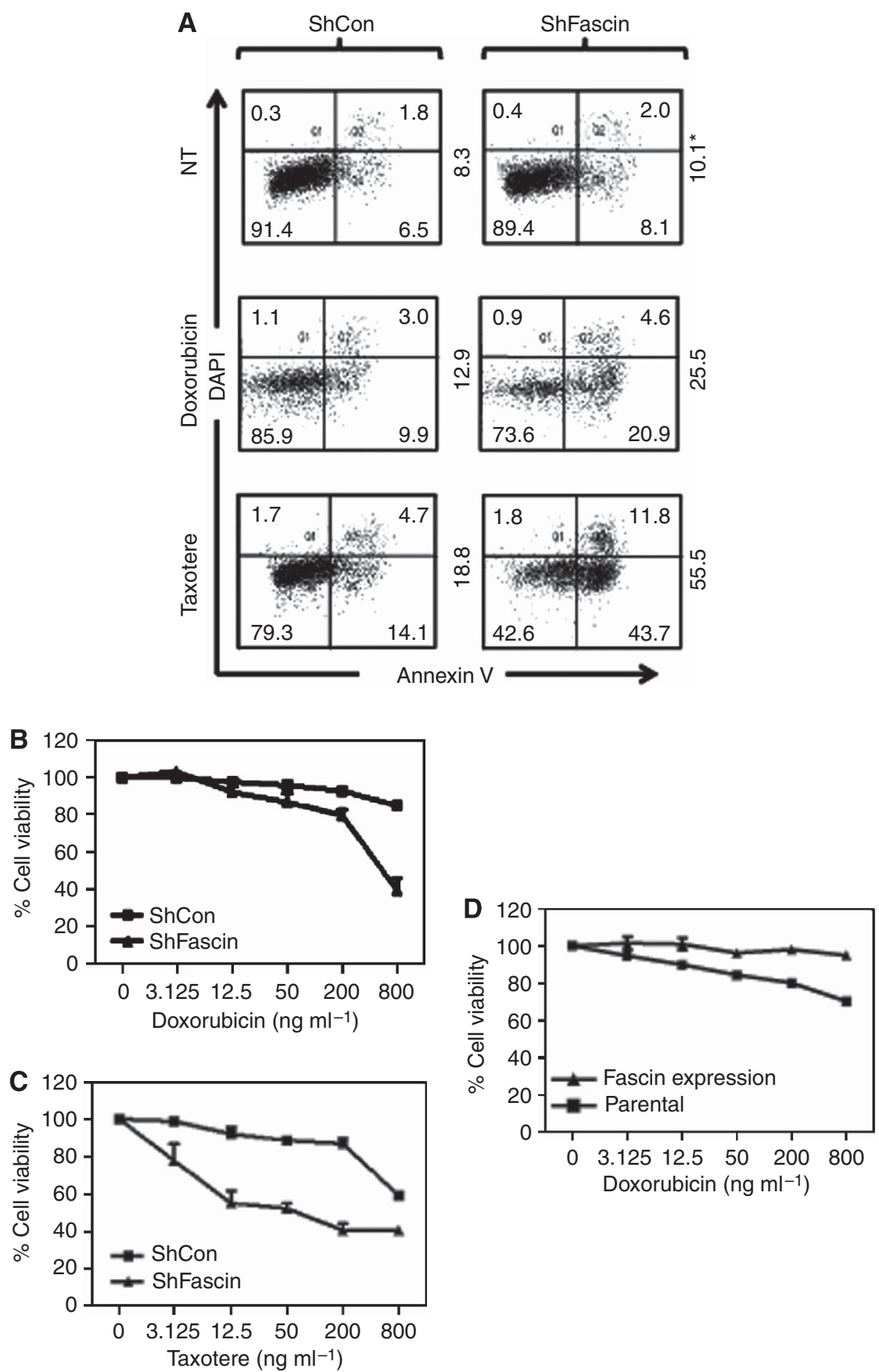

Figure 3. Fascin-positive breast cancer cells are more resistant to chemotherapy-mediated apoptosis. (A) Show representative dot plot of Annexin $\mathrm{V}$ and DAPI staining following treatment of ShCon (top) or ShFascin (bottom) breast cancer cells without (left) or with $200 \mathrm{ng} \mathrm{ml} \mathrm{l}^{-1}$ of doxorubicin (middle) or taxotere (bottom) for $24 \mathrm{~h}$. ${ }^{*}$ Numbers on the right of the dot plot represent the total percentage of apoptosis (Q2 + Q4). Bar graphs showing mean \pm s.d. viable cells following treatment with increased doses of either doxorubicin (B) or taxotere (C) and data is representative of triplicate experiments. (D) Bar graphs showing mean \pm s.d. viable cells of triplicate experiments following treatment of T47-D cells with doxorubicin. T47-D is the parental T47-D cells, which are fascin negative. Fascin expression are T47-D cells that expressed wild-type fascin.

responses in vitro. Both fascin-positive and -negative cells showed a dose-dependent apoptotic cell death upon exposure to doxorubicin (Figure 3A and B). However, fascin-negative cells were more sensitive to chemotherapy-mediated apoptosis than fascin-positive cells. Similar results (Figure $3 \mathrm{~A}$ and C) were observed when we used taxotere, another chemotherapeutic agent that is commonly used with or without doxorubicin to treat breast cancer patients (Kaushal et al, 2004; Minotti et al, 2004). This data has been confirmed using another fascin-specific SiRNA and by fascin overexpression (Supplementary Figure 1). To further confirm the direct link between fascin expression and chemoresistance, we used T47-D, a fascin-negative breast cancer cell line, where we have expressed fascin in them as previously described (Al-Alwan et al, 2011). Interestingly, T47-D cells expressing fascin showed more resistance to chemotherapy-mediated apoptosis compared with parental cells (Figure 3D). Similarly, expression of fascin in the fascin-negative SK-BR-3 breast cancer cell line confer chemoresistance (data not shown). Altogether, our loss and gain of function data demonstrated that fascin expression in breast cancer cells is involved in their chemoresistance. 
Chemoresistance of fascin-positive breast cancer cells is at least partially mediated via increased PI3K/Akt activation paralleled by enhanced anti-apoptotic and reduced proapoptotic genes. Our data clearly demonstrated fascin involvement in conferring resistance to chemotherapy-mediated apoptosis in breast cancer cells. To elucidate the underlying mechanism, we have tested whether fascin expression in breast cancer cells modify the expression of the apoptotic genes and/or its upstream regulators. It has be reported that many cancer cells can evade apoptosis through the activation of phosphatidylinositol 3-kinase (PI3K)/Akt pathway and mutation or downregulation of tumour suppressor gene PTEN, a negative regulator of the PI3K pathway (West et al, 2002; Fresno Vara et al, 2004; Zhang \& Yu, 2010; Dituri et al, 2011). We thus asked if the lack of fascin expression in breast cancer cells sensitises them to apoptosis via downregulation of the PI3K pathway. Treatment of fascin-positive breast cancer cells with
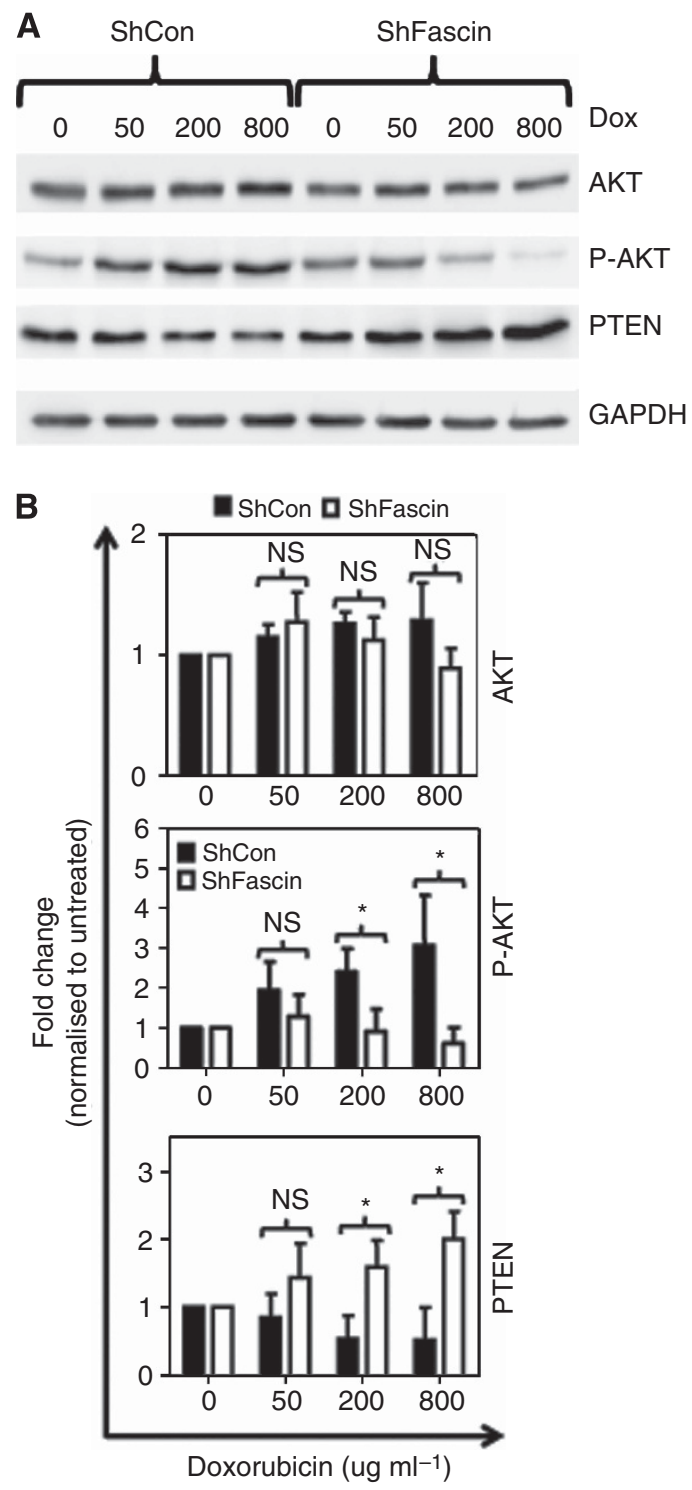

Figure 4. Doxorubicin induces phosphorylated AKT and represses PTEN expression in fascin-positive breast cancer cells. (A) Western blot showing the expression levels of Akt, p-Akt and PTEN in ShCon and ShFascin cells following treatment with various doses of doxorubicin for $24 \mathrm{~h}$. (B) Bar graph showing quantitation of total, p-Akt and PTEN in ShCon and ShFascin cells after treatment with various doses of doxorubicin. Results showed the mean of triplicate experiments after normalisation to GAPDH and each time point is normalised to 0 time. ${ }^{\star} P<0.05$. doxorubicin (Figure 4) or taxotere (Supplementary Figure 2) induced increase in the AKT phosphorylation as well as the total AKT levels in a dose-dependent manner. Moreover, expression of PTEN in the fascin-positive cells was noticeably suppressed upon chemotherapy treatment. On the contrary, fascin-negative cells showed reduced AKT phosphorylation and enhanced PTEN expression after chemotherapy. The link between fascin expression and enhanced PI3K signalling was also observed in fascinexpressing T47-D cells after drug treatment when compared with parental group (Supplementary Figure 3). In fact, fascin appears to confer chemoresistance mainly through enhancing the PI3K signalling as demonstrated by upregulation of Akt phosphorylation. Indeed, inhibition of Akt pathway using the AKT IV selective inhibitor (Figure 5A), eliminated the differences in chemotherapy-mediated apoptotic cell death between fascinpositive and -negative cells (Figure 5B). These data demonstrated a strong association between fascin expression and enhancement of PI3K signalling in response to chemotherapy, as reflected by the increased expression of AKT phosphorylation and decreased expression of the PTEN in the fascin-positive group.

$\mathrm{X}$-linked inhibitor of apoptosis protein (XIAP) is one of the inhibitor of apoptosis protein (IAP) family that was previously reported to be a downstream target of Akt (Dan et al, 2004). We thus tested whether fascin-mediated enhanced PI3K signalling resulted in increased expression of XIAP or any other member of the IAP family. Among the five IAP tested genes, only XIAP and Livin showed noticeable increase in fascin-positive cells after doxorubicin (Figure 6A and B) or taxotere (Supplementary Figure $4 \mathrm{~A}$ and $\mathrm{B}$ ) treatment in a dose-dependent manner. In the fascin-negative group, the expression levels of XIAP and Livin were sharply dropped especially following high dose of doxorubicin treatment. Given that our data showed increased expression of IAP genes in fascin-positive cells, we asked if this observation correlated
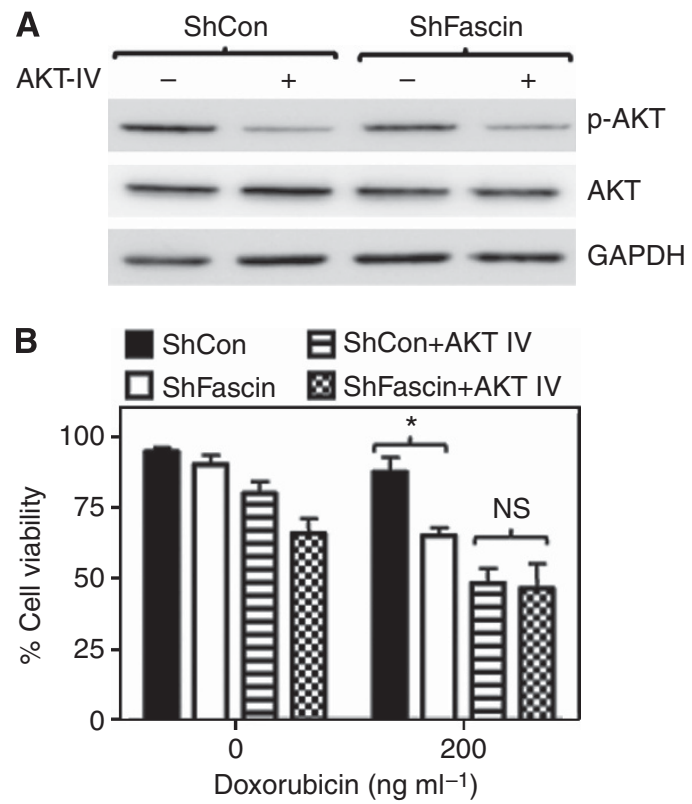

Figure 5. Resistance of fascin-positive cells to apoptosis is primarily mediated via the Akt pathway. (A and B) ShCon or ShFascin breast cancer cells were pre-incubated in the presence or absence of AKT IV inhibitor $(2 \mu \mathrm{M})$ for $24 \mathrm{~h}$ followed by treatment with $200 \mathrm{ng} \mathrm{ml}^{-1}$ of doxorubicin for $48 \mathrm{~h}$. (A) Western blot showing the expression levels of Akt, p-Akt and GAPDH in ShCon and ShFascin cells following treatment with AKT IV inhibitor. (B) Cells were harvested and apoptotic cell death was determined as above. Results showing mean \pm s.d. viable cells following treatment with doxorubicin and data is representative of triplicate experiments. ${ }^{\star} P<0.05$. 


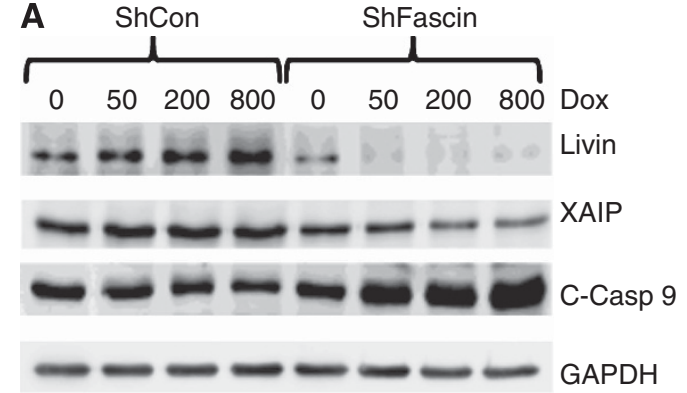

B

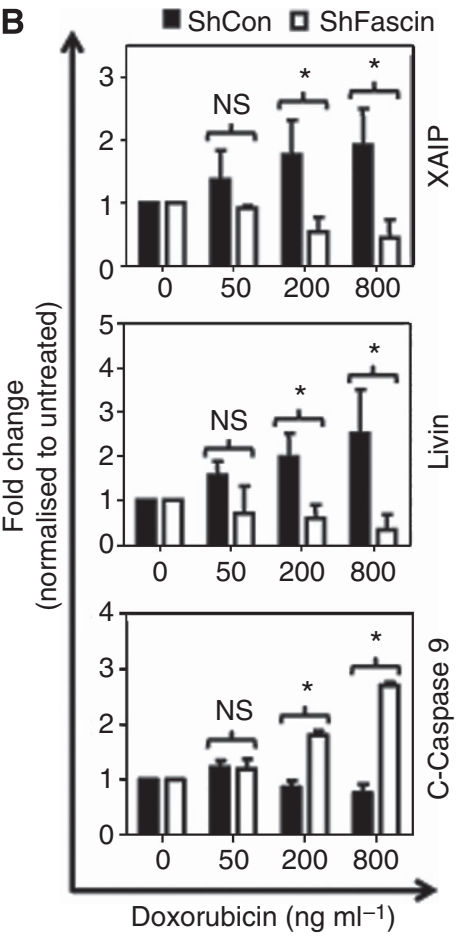

Figure 6. Doxorubicin induces XIAP, Livin and represses caspase 9 expression in fascin-positive breast cancer cells. (A) Western blot showing the expression levels of XIAP, Livin and caspase 9 in ShCon and ShFascin cells following treatment with various doses of doxorubicin for $24 \mathrm{~h}$. (B) Bar graph showing quantitation of XIAP, Livin and caspase 9 in ShCon and ShFascin cells after treatment with various doses of doxorubicin. Results showed the mean of triplicate experiments after normalisation to GAPDH and each time point is normalised to 0 time. ${ }^{\star} P<0.05$.

with decreased expression of caspases, substrates of apoptosis. XIAP was reported to have a direct effect on caspase 9, which is involved in the initiation of the intrinsic apoptotic pathway and caspase 3, which is critical for the execution of apoptosis (Dan et al, 2004) and Poly (ADP-ribose) polymerase (PARP), one of the main cleavage targets of caspase 3 that facilitate cellular death upon cleavage. In contrast to the increased levels of IAP, the expression of the active forms of caspase 9 (Figure 6) and caspase 3 and cleaved form of PARP (Figure 7A and B and Supplementary Figure 5) was significantly reduced in fascin-positive cells following doxorubicin treatment. Similar results were observed following the treatment with taxotere (Supplementary Figure 6). Collectively, our data showed that fascin-mediated chemoresistance in breast cancer cells is associated with upregulation of PI3K/Akt signalling and IAP gene expression; and downregulation of the proapoptotic genes.

Fascin-mediated chemoresistance in breast cancer cells via regulation of FAK phosphorylation. It was previously reported
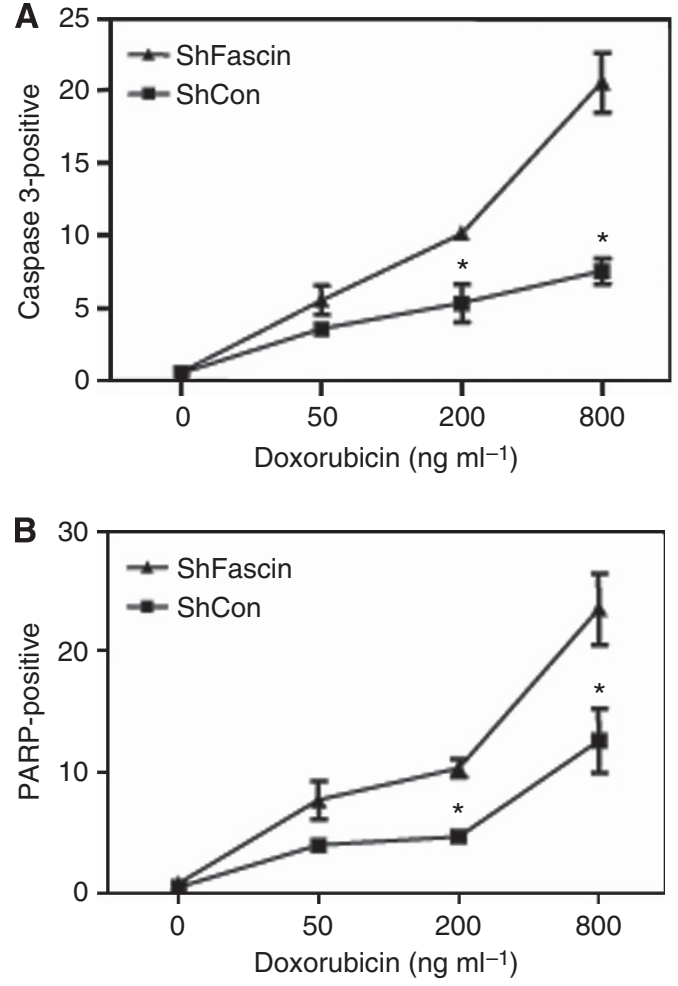

Figure 7. Doxorubicin suppresses the expression of caspase 3 and PARP in fascin-positive breast cancer cells. (A) Graph showing the percentage of active form of caspase 3 expressing cells in ShCon or ShFascin group following the treatment with doxorubicin for $24 \mathrm{~h}$.

(B) Graph showing percentage of cleaved PARP-positive cells in ShCon or ShFascin group following the treatment with doxorubicin for $24 \mathrm{~h}$.

Data are expressed as mean \pm s.d. positive cells and are representative of triplicate experiments. ${ }^{\star} P<0.05$.

that focal adhesion kinase (FAK) can regulate integrin-mediated survival in epithelial cells mainly through the PI3K/AKT pathway (Chen \& Guan, 1994; Khwaja et al, 1997; Xia et al, 2004). Here we evaluated the involvement of FAK activation in fascin-mediated $\mathrm{PI} 3 \mathrm{~K} / \mathrm{AKT}$ signalling pathway in our system. In a similar pattern to the AKT phosphorylation (Figure 4), treatment with doxorubicin (Figure $8 \mathrm{~A}$ and $\mathrm{B}$ ) or taxotere (Supplementary Figure 7A and B) induced a dose-dependent increase in FAK phosphorylation of fascin-positive breast cancer cells as compared with their fascinnegative counterparts. Contrary to the increase in the level of FAK phosphorylation following treatment, there was an increase in the total FAK levels at lower dose with significant decline at the higher dose of drug.

To test whether fascin confer chemoresistance predominantly via enhancing FAK phosphorylation, we inhibited FAK expression using selective inhibitor and examined its effect on apoptosis. Interestingly, inhibition of FAK eliminated the difference in chemotherapy-mediated apoptotic cell death between fascinpositive and -negative cells (Figure $8 \mathrm{C}$ ). Altogether, our data demonstrate that the lack of fascin expression sensitises cells to chemotherapy-mediated apoptosis via regulating the activation of FAK, a known pathway to regulate drug resistance via the AKT pathway.

\section{DISCUSSION}

Despite success in the treatment of primary breast cancer, the outcome remains poor for some types of breast cancers including locally advanced and metastatic breast cancers (Khope et al, 1988). 


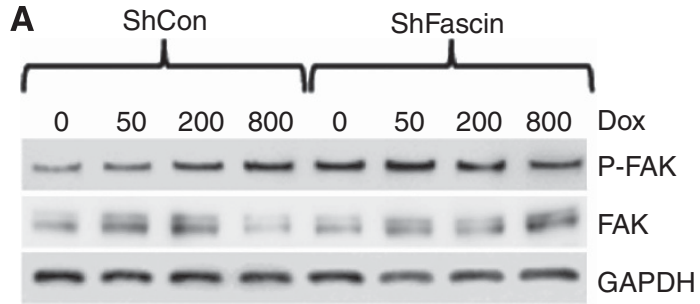

B

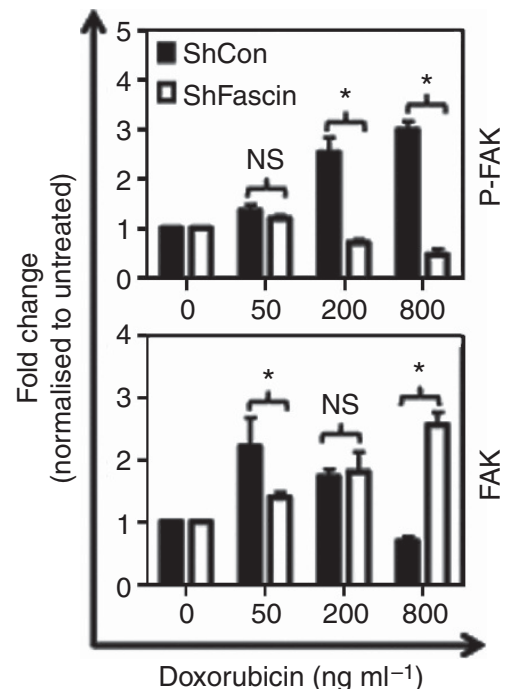

C

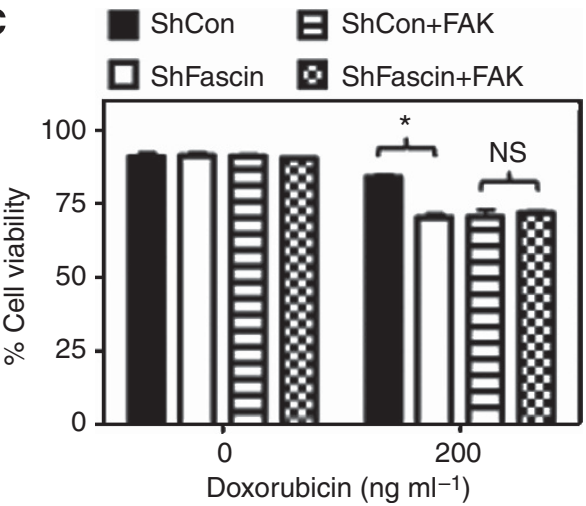

Figure 8. Fascin knockdown breast cancer cells exhibits suppressed doxorubicin-mediated FAK phosphorylation. (A) Western blot showing the expression levels of FAK, p-FAK and GAPDH in ShCon and ShFascin cells following the treatment with various doses of doxorubicin for $24 \mathrm{~h}$. (B) Bar graph showing quantitation of total, p-FAK in ShCon and ShFascin cells after treatment with various doses of doxorubicin. Results showed the mean of triplicate experiments after normalisation to GAPDH and each time point is normalised to 0 time. (C) ShCon or ShFascin breast cancer cells were pre-incubated in the presence or absence of FAK inhibitor $(1 \mu \mathrm{m})$ for $8 \mathrm{~h}$ followed by the treatment with $200 \mathrm{ng} \mathrm{ml}^{-1}$ of doxorubicin for $48 \mathrm{~h}$. Cells were harvested and apoptotic cell death was determined as above.

Results showing mean \pm s.d. viable cells following the treatment with doxorubicin and data are representative of triplicate experiments. ${ }^{\star} P<0.05$.

While current treatment targets the bulk of tumour cells, some cancer cells manage to escape killing by chemotherapy. It is strongly believed that these drug-resistant cancer cells can subsequently reestablish the tumour and lead to metastasis (Khope et al, 1988). In this study, we have demonstrated a role for the expression of fascin in breast cancer cells in regulating their chemoresistance. Fascin involvement in this process appears to be mediated at least in part via enhancement of the PI3K/Akt signalling and upregulation of anti-apoptotic genes paralleled with suppression of the proapoptotic genes, thus reducing apoptosis.

In the previous study, we have demonstrated a significant correlation between increased fascin expression in breast cancer patients with bad prognostic markers and shorter survival (AlAlwan et al, 2011). Reduced fascin expression strongly correlated with enhanced expression of BRMS1, which has been shown to be a critical regulator of breast cancer metastasis (Samant et al, 2006). Interestingly, we found fascin to be associated with enhanced NF$\kappa \mathrm{b}$ signalling and upregulation of the metastasis-associated genes (Al-Alwan et al, 2011). The relationship between fascin expression and poor prognostic markers, especially metastasis, strongly suggests a potential role for this protein in regulating some elements of cancer cell resistance to therapy. This assumption was based on the fact that chemoresistant cancer cells are responsible for tumour relapse and metastasis (Khope et al, 1988). Consistent with our hypothesis, fascin-positive breast cancer patients that received adjuvant therapy had shorter survival when compared with the fascin-negative counterparts. These findings implied the involvement of fascin expression in regulating chemoresistance although other confounding factors might be involved in this process like hormone receptor status, which we have previously demonstrated to highly correlate with fascin (Al-Alwan et al, 2011). To resolve this concern, we used xenograft animal model and in vitro tests. Interestingly, fascin-negative MDA-MB-231 tumour xenografts were more sensitised to chemotherapy, demonstrating a direct involvement of fascin in regulating chemoresistance. Furthermore, both MDA-MB-231 and T47-D, which are oestrogen receptor negative and positive, respectively, responded to chemotherapy in a fascin expression-dependent manner irrespective of their oestrogen receptor status. Altogether, these results supported the specific role of fascin in regulating chemoresistance and recommend the evaluation of its expression for future treatment of metastatic breast cancer patients. Since this group of fascin-positive patients is not responding to the chemotherapy, it might be more effective to include fascintargeting agents for a better treatment outcome. Along this line of thought, synthetic migrastatin analogue was demonstrated to potently inhibit breast cancer metastasis to the lung by specifically targeting fascin (Chen et al, 2010).

Better understanding of the underlying mechanism of fascin regulation of breast cancer metastasis and chemoresistance will have an impact on developing treatment regiments that control fascin upstream or downstream targets. Many types of cancers including breast cancer were reported to express constitutive activation of the PI3K/Akt pathway, which is also involved in regulating many cellular processes including survival, growth and motility (reviewed in West et al (2002)). In addition to the amplified activation of PI3K/Akt pathway, many cancer cells including breast were reported to have deletion or mutation of PTEN, one of the main negative regulators of the PI3K/Akt pathway. Inhibition of the PI3K/Akt pathway using the global inhibitor (LY294002) sensitised many types of tumour cells to apoptotic cell death by doxorubicin (Fujiwara et al, 2006). Our findings that fascin expression is associated with enhanced PI3K/ Akt activation and downregulation of its negative regulator (PTEN) may explain the resistance of the fascin-positive cells to chemotherapy. The findings by Alam et al (2012) where they demonstrated enhanced AKT phosphorylation in oral squamous carcinoma cells that over-expressed fascin further support fascinmediated regulation of AKT phosphorylation. In fact, our results showed that when the Akt pathway was selectively inhibited using Akt IV inhibitor, the levels of doxorubicin-mediated apoptotic cell death were similar between fascin-positive and -negative cells. This data strongly suggest that fascin executes its anti-apoptotic effect in cancer cells, at least in part, via the PI3K/Akt pathway. 
It has been well established that Akt confers its anti-apoptotic role through the phosphorylation of various downstream targets (Nicholson and Anderson, 2002; Fresno Vara et al, 2004; Sansal and Sellers, 2004). One of these downstream targets is the phosphorylation of NF- $\kappa \mathrm{B}$, a pathway that we have previously identified to be enhanced in fascin-positive breast cancer cells (AlAlwan et al, 2011). Indeed, Akt has been reported to phosphorylate XIAP, a process that protected XIAP from degradation in response to cisplatin treatment and inhibited apoptosis (Dan et al, 2004). Consistent with this finding, our results showed that fascinmediated chemoresistance in breast cancer cells were associated with sustained/enhanced levels of XIAP and Livin. Both proteins were reduced in chemosensitive fascin-negative breast cancer cells. It is important to note that among the IAP family protein, XIAP has been identified as the most potent caspase inhibitor (Holcik et al, 2001). This is in line with our findings that showed inhibition of caspase 3 and 9 activation and reduced levels of cleaved PARP in fascin-positive chemoresistant breast cancer cells. Our data clearly demonstrated that fascin expression in the chemoresistant breast cancer cells was consistent with enhanced PI3K/Akt, increased IAP and reduced levels of anti-apoptotic genes. Our results are consistent with previous study that demonstrated enhanced AKT phosphorylation in oral squamous carcinoma cells that overexpressed fascin (Alam et al, 2012). This expression profile demonstrates a role for these proteins in helping fascin to confer its pro-survival effect in breast cancer cells.

Focal adhesion kinase is another factor that has been reported to regulate integrin-mediated survival in epithelial cells mainly through the PI3K/AKT pathway (Chen \& Guan, 1994; Khwaja et al, 1997; Xia et al, 2004). Upon integrin ligation, FAK is recruited and activated by phosphorylation - a step that creates a binding site for the PI3K/AKT subunit (Chen et al, 1996; Schlaepfer and Hunter, 1998; Eliceiri et al, 2002). It was proposed that fascin regulates migration and MMP-2 secretion by human hepatic stellate cells via the FAK-AKT pathway (Uyama et al, 2012). In addition, beta 1 integrin was reported to associate with activated PKC-alpha, a kinase that also interacts with fascin in mouse skeletal myoblastic cells (C2C12; Anilkumar et al, 2003). Our data indicated decreased doxorubicin-mediated FAK phosphorylation in fascin knockdown cells. Fascin expression in breast cancer cells may confer resistance to chemotherapy-mediated apoptosis via regulating the phosphorylation of FAK, a pathway known to regulate drug resistance via the AKT pathway (Adams, 2004). Surprisingly, the baseline levels of FAK phosphorylation in fascin-negative cells were elevated. It is possible that in the absence of fascin some compensatory mechanisms induced FAK phosphorylation in an effort to rescue cells from apoptosis. However, the exposure of fascin-negative cells to chemotherapy did not induce further FAK phosphorylation as compared with the fascinpositive cells. This data showed that FAK phosphorylation alone was not enough to protect cells from chemotherapy-mediated killing in the absence of fascin. Furthermore, the response of cells to chemotherapy in fascin-positive cells was not solely dependent on FAK phosphorylation. While FAK phosphorylation as a response to other cellular challenges may not solely be controlled by fascin, the differential response to drug treatment between the fascin-positive and -negative group was eliminated when FAK was selectively inhibited. Fascin may lead to stabilisation and accumulation of phospho-FAK in the cells and this step becomes critical in the presence of chemotherapy. Furthermore, fascinmediated cytoskeletal rearrangements may provide a platform that facilitates the interaction of phospho-FAK with other mediators that are responsible for conferring the chemoresistance.

In conclusion, this study identified fascin to have a key role in regulating breast cancer chemoresistance and dissected the underlying mechanism. This suggests that for an efficient treatment of breast cancer in which fascin is expressed, new chemotherapeutic strategies should be considered. Drug screening, which aims at finding compounds that can specifically and efficiently target fascin or its downstream effectors, might prove to be a successful strategy. Combinational therapy, such as anti-fascin-specific compounds and/or inhibition of the PI3K/AKT, in addition to the chemotherapy might be another potential strategy.

\section{ACKNOWLEDGEMENTS}

We are grateful to the administration of Research Centre and Research Affair Council for their support. We are grateful to Dr Josephine C Adam for providing the fascin-GFP fusion constructs, Mrs Pulicat Manogaran and Amer Almazrou for helping in the analysis of FACS data. This work was supported by the Research Advisory Council (proposal grant 2060 016), King Faisal Specialist Hospital and Research Centre. The funders had no role in study design, data collection and analysis, decision to publish, or preparation of the manuscript.

\section{AUTHOR CONTRIBUTIONS}

MAA conceived and designed the experiments. HG, SA-K, SO and $\mathrm{RB}$ performed the in vitro experiments. $\mathrm{AD}$ and $\mathrm{FM}$ performed the animal experiments. AT, TA-T and DA analysed the data. HG and MAA wrote the paper.

\section{REFERENCES}

Adams JC (2004) Roles of fascin in cell adhesion and motility. Curr Opin Cell Biol 16(5): 590-596.

Al-Alwan M, Olabi S, Ghebeh H, Barhoush E, Tulbah A, Al-Tweigeri T, Ajarim D, Adra C (2011) Fascin is a key regulator of breast cancer invasion that acts via the modification of metastasis-associated molecules. PLoS One 6(11): e27339.

Al-Tweigeri TA, Ajarim DS, Alsayed AA, Rahal MM, Alshabanah MO, Tulbah AM, Al-Malik OA, Fatani DM, El-Husseiny GA, Elkum NB, Ezzat AA (2010) Prospective phase II study of neoadjuvant doxorubicin followed by cisplatin/docetaxel in locally advanced breast cancer. Med Oncol 27(3): 571-577.

Alam H, Bhate AV, Gangadaran P, Sawant SS, Salot S, Sehgal L, Dange PP, Chaukar DA, D'Cruz AK, Kannanl S, Gude R, Kane S, Dalal SN, Vaidya MM (2012) Fascin overexpression promotes neoplastic progression in oral squamous cell carcinoma. BMC Cancer 12: 32.

Anilkumar N, Parsons M, Monk R, Ng T, Adams JC (2003) Interaction of fascin and protein kinase Calpha: a novel intersection in cell adhesion and motility. EMBO J 22(20): 5390-5402.

Balduzzi A, Bagnardi V, Rotmensz N, Dellapasqua S, Montagna E, Cardillo A, Viale G, Veronesi P, Intra M, Luini A, Pruneri G, Mastropasqua G, Goldhirsch A, Colleoni M (2013) Survival outcomes in breast cancer patients with low estrogen/progesterone receptor expression. Clin Breast Cancer 14(4): 258-264.

Chen HC, Appeddu PA, Isoda H, Guan JL (1996) Phosphorylation of tyrosine 397 in focal adhesion kinase is required for binding phosphatidylinositol 3-kinase. J Biol Chem 271(42): 26329-26334.

Chen HC, Guan JL (1994) Association of focal adhesion kinase with its potential substrate phosphatidylinositol 3-kinase. Proc Natl Acad Sci USA 91(21): 10148-10152.

Chen L, Yang S, Jakoncic J, Zhang JJ, Huang XY (2010) Migrastatin analogues target fascin to block tumour metastasis. Nature 464(7291): 1062-1066.

Dan HC, Sun M, Kaneko S, Feldman RI, Nicosia SV, Wang HG, Tsang BK, Cheng JQ (2004) Akt phosphorylation and stabilization of X-linked inhibitor of apoptosis protein (XIAP). J Biol Chem 279(7): 5405-5412.

Dellapasqua S, Bagnardi V, Balduzzi A, Iorfida M, Rotmensz N, Santillo B, Viale G, Ghisini R, Veronesi P, Luini A, Morra A, Goldhirsch A, Colleoni M (2014) Outcomes of patients with breast cancer who present with ipsilateral supraclavicular or internal mammary lymph node metastases. Clin Breast Cancer 14(1): 53-60. 
Dituri F, Mazzocca A, Giannelli G, Antonaci S (2011) PI3K functions in cancer progression, anticancer immunity and immune evasion by tumors. Clin Dev Immunol 2011: 947858.

Duh FM, Latif F, Weng Y, Geil L, Modi W, Stackhouse T, Matsumura F, Duan DR, Linehan WM, Lerman MI et al. (1994) cDNA cloning and expression of the human homolog of the sea urchin fascin and Drosophila singed genes which encodes an actin-bundling protein. DNA Cell Biol 13(8): 821-827.

Eliceiri BP, Puente XS, Hood JD, Stupack DG, Schlaepfer DD, Huang XZ, Sheppard D, Cheresh DA (2002) Src-mediated coupling of focal adhesion kinase to integrin alpha(v)beta5 in vascular endothelial growth factor signaling. J Cell Biol 157(1): 149-160.

Fresno Vara JA, Casado E, de Castro J, Cejas P, Belda-Iniesta C, Gonzalez-Baron M (2004) PI3K/Akt signalling pathway and cancer. Cancer Treat Rev 30(2): 193-204.

Fujiwara Y, Kawada K, Takano D, Tanimura S, Ozaki K, Kohno M (2006) Inhibition of the PI3 kinase/Akt pathway enhances doxorubicin-induced apoptotic cell death in tumor cells in a p53-dependent manner. Biochem Biophys Res Commun 340(2): 560-566.

Ghebeh H, Mohammed S, Al-Omair A, Qattan A, Lehe C, Al-Qudaihi G, Elkum N, Alshabanah M, Bin Amer S, Tulbah A, Ajarim D, Al-Tweigeri T, Dermime S (2006) The B7-H1 (PD-L1) T lymphocyte-inhibitory molecule is expressed in breast cancer patients with infiltrating ductal carcinoma: correlation with important high-risk prognostic factors. Neoplasia 8(3): 190-198.

Gladney CD, Martinez VG, Brumbaugh KA, DeGroot BJ, Linville RC, Oommen AM, Huebinger RM, Messer LA, Allan MF, Pomp D (1999) Mapping of the Prostaglandin-Endoperoxide Synthase 2 (PTGS2) gene to porcine chromosome 9 and bovine chromosome 16 by linkage analysis using novel PCR-RFLP. J Anim Sci 77(3): 787-788.

Hirsch HA, Iliopoulos D, Tsichlis PN, Struhl K (2009) Metformin selectively targets cancer stem cells, and acts together with chemotherapy to block tumor growth and prolong remission. Cancer Res 69(19): 7507-7511.

Holcik M, Gibson H, Korneluk RG (2001) XIAP: apoptotic brake and promising therapeutic target. Apoptosis 6(4): 253-261.

Jiang P, Enomoto A, Takahashi M (2009) Cell biology of the movement of breast cancer cells: intracellular signalling and the actin cytoskeleton. Cancer Lett 284(2): 122-130.

Kaushal GP, Liu L, Kaushal V, Hong X, Melnyk O, Seth R, Safirstein R, Shah SV (2004) Regulation of caspase-3 and -9 activation in oxidant stress to RTE by forkhead transcription factors, Bcl-2 proteins, and MAP kinases. Am J Physiol Renal Physiol 287(6): F1258-F1268.

Khope S, Rao PL, Oommen AN (1988) Primary lymphomatous stricture of ileum. Indian Pediatr 25(3): 294-296.

Khwaja A, Rodriguez-Viciana P, Wennstrom S, Warne PH, Downward J (1997) Matrix adhesion and Ras transformation both activate a phosphoinositide 3-OH kinase and protein kinase B/Akt cellular survival pathway. EMBO J 16(10): 2783-2793.

Minotti G, Menna P, Salvatorelli E, Cairo G, Gianni L (2004) Anthracyclines: molecular advances and pharmacologic developments in antitumor activity and cardiotoxicity. Pharmacol Rev 56(2): 185-229.
Mosialos G, Birkenbach M, Ayehunie S, Matsumura F, Pinkus GS, Kieff E, Langhoff E (1996) Circulating human dendritic cells differentially express high levels of a 55-kd actin-bundling protein. Am J Pathol 148(2): 593-600.

Mosialos G, Yamashiro S, Baughman RW, Matsudaira P, Vara L, Matsumura F, Kieff E, Birkenbach M (1994) Epstein-Barr virus infection induces expression in B lymphocytes of a novel gene encoding an evolutionarily conserved 55-kilodalton actin-bundling protein. $J$ Virol 68(11): 7320-7328.

Naffar-Abu-Amara S, Shay T, Galun M, Cohen N, Isakoff SJ, Kam Z, Geiger B (2008) Identification of novel pro-migratory, cancer-associated genes using quantitative, microscopy-based screening. PLoS One 3(1): e1457.

Nicholson KM, Anderson NG (2002) The protein kinase B/Akt signalling pathway in human malignancy. Cell Signal 14(5): 381-395.

Pinkus GS, Pinkus JL, Langhoff E, Matsumura F, Yamashiro S, Mosialos G, Said JW (1997) Fascin, a sensitive new marker for Reed-Sternberg cells of hodgkin's disease. Evidence for a dendritic or B cell derivation? Am J Pathol 150(2): 543-562.

Rochefort H, Platet N, Hayashido Y, Derocq D, Lucas A, Cunat S, Garcia M (1998) Estrogen receptor mediated inhibition of cancer cell invasion and motility: an overview. J Steroid Biochem Mol Biol 65(1-6): 163-168.

Samant RS, Debies MT, Hurst DR, Moore BP, Shevde LA, Welch DR (2006) Suppression of murine mammary carcinoma metastasis by the murine ortholog of breast cancer metastasis suppressor 1 (Brms1). Cancer Lett 235(2): 260-265.

Sansal I, Sellers WR (2004) The biology and clinical relevance of the PTEN tumor suppressor pathway. J Clin Oncol 22(14): 2954-2963.

Schlaepfer DD, Hunter T (1998) Integrin signalling and tyrosine phosphorylation: just the FAKs? Trends Cell Biol 8(4): 151-157.

Tong D, Czerwenka K, Sedlak J, Schneeberger C, Schiebel I, Concin N, Leodolter S, Zeillinger R (1999) Association of in vitro invasiveness and gene expression of estrogen receptor, progesterone receptor, pS2 and plasminogen activator inhibitor-1 in human breast cancer cell lines. Breast Cancer Res Treat 56(1): 91-97.

Uyama N, Iimuro Y, Kawada N, Reynaert H, Suzumura K, Hirano T, Kuroda N, Fujimoto J (2012) Fascin, a novel marker of human hepatic stellate cells, may regulate their proliferation, migration, and collagen gene expression through the FAK-PI3K-Akt pathway. Lab Invest 92(1): 57-71.

West KA, Castillo SS, Dennis PA (2002) Activation of the PI3K/Akt pathway and chemotherapeutic resistance. Drug Resist Updat 5(6): 234-248.

Xia H, Nho RS, Kahm J, Kleidon J, Henke CA (2004) Focal adhesion kinase is upstream of phosphatidylinositol 3-kinase/Akt in regulating fibroblast survival in response to contraction of type I collagen matrices via a beta 1 integrin viability signaling pathway. J Biol Chem 279(31): 33024-33034.

Zhang S, Yu D (2010) PI(3)king apart PTEN's role in cancer. Clin Cancer Res 16(17): 4325-4330.

(c) (5) (2) This work is licensed under the Creative Commons Attribution-NonCommercial-Share Alike 3.0 Unported License. To view a copy of this license, visit http://creativecommons. org/licenses/by-nc-sa/3.0/

Supplementary Information accompanies this paper on British Journal of Cancer website (http://www.nature.com/bjc) 